0092-8240(94)00047-6

\title{
TOPOLOGICAL AND PHENOMENOLOGICAL CLASSIFICATION OF BURSTING OSCILLATIONS
}

\author{
- RICHARD BERTRAM,$\uparrow$ MANISH J. BUTTE, $\ddagger$ TIM KIEMEL $\uparrow \S$ \\ and ARTHUR SHERMAN $\dagger$ \\ †National Institutes of Health, \\ National Institutes of Diabetes and Digestive and Kidney Diseases, \\ Mathematical Research Branch, \\ BSA Building, Suite 350, \\ Bethesda, MD 20814, U.S.A. \\ (E.mail: rbertram@helix.nih.gov; sherman@helix.nih.gov) \\ $\ddagger$ Brown University, \\ Box G-8090, \\ Providence, RI 02912, U.S.A. \\ (E. mail: manish_butte@brown.edu) \\ $\S$ Department of Zoology, \\ University of Maryland, \\ College Park, MD 20742, U.S.A. \\ (E.mail:kiemel@swimmers.umd.edu)
}

We describe a classification scheme for bursting oscillations which encompasses many of those found in the literature on bursting in excitable media. This is an extension of the scheme of Rinzel (in Mathematical Topics in Population Biology, Springer, Berlin, 1987), put in the context of a sequence of horizontal cuts through a two-parameter bifurcation diagram. We use this to describe the phenomenological character of different types of bursting, addressing the issue of how well the bursting can be characterized given the limited amount of information often available in experimental settings.

1. Introduction. Bursting is an oscillation in which an observable of the system, such as voltage or chemical concentration, changes periodically between an active phase of rapid spike oscillations and a phase of quiescence. Since bursting was reported in the electrical activity of neuron $R_{15}$ of the abdominal ganglion of Aplysia (Strumwasser, 1967; Alving, 1968) it has been found to be the primary mode of electrical behavior in many nerve and endocrine cells. Examples include thalamic neurons (Deschênes et al., 1982; Crunelli et al., 1987), hippocampal pyramidal neurons (Wong and Prince, 1981), AB neurons (Harris-Warrick and Flamm, 1987), dopaminergic neurons 
of the mammalian midbrain (Johnson et al., 1992), and pancreatic $\beta$-cells (Dean and Mathews, 1970; Ashcroft and Rorsman, 1989). In addition, bursting oscillations have been observed in chemical systems such as the BelousovZhabotinsky reaction (Hudson et al., 1979).

Numerous models of bursting have been developed (e.g. Plant and Kim, 1976; Canavier et al., 1991; Bertram, 1993; Smolen and Keizer, 1992; Guckenheimer et al., 1994; Rinzel and Troy, 1982; Traub et al., 1991; Wang et $a l ., 1991)$ and a systematic mathematical analysis of one such model was first performed by Rinzel (1985). In this analysis the system variables were classified as either "fast", if the variable changed significantly over the duration of a single spike, or "slow", if significant change occurred only over the duration of the burst. The bursting oscillation is generated as the evolution of the slow variables switches the fast dynamics between steady state and oscillatory dynamics. Analysis of the bursting is carried out by studying the solution structure or topology of the fast subsystem, with the slow variables treated as slowly varying parameters.

In the classification scheme of Rinzel (1987) three types of bursting oscillations were described (Table 1). We now concentrate primarily on the phenomenological aspects of these oscillations, discussing the topological structure in subsequent sections. In type I bursting the frequency of spiking decreases monotonically through the active phase (Fig.1A). The monotonic spike frequency profile is due to a single passage of the bursting trajectory through a saddle-loop bifurcation, at the end of the active phase. Also, no spike

Table 1. Classification scheme first described by Rinzel (1987) and discussed in terms of a generic sequence of bifurcations in Fig. 7 in the present paper. $\mathrm{SN}=$ saddle node;

SNP $=$ saddle node of periodics; $\mathrm{SL}=$ saddle loop; SNIC $=$ saddle node on an invariant circle; $\mathrm{UH}=$ unstable (subcritical) Hopf

\begin{tabular}{cll}
\hline & \multicolumn{2}{c}{ Burst classification scheme } \\
\hline Type & \multicolumn{1}{c}{ Topology } & \multicolumn{1}{c}{ Phenomenology } \\
\hline I & $\begin{array}{l}\text { Active phase begins at SN } \\
\text { Active phase ends at SL } \\
\text { Fast subsystem bistable } \\
\end{array}$ & Spike frequency monotonic \\
& 1 Slow variable sufficient & Can be reset by brief perturbation \\
II & $\begin{array}{l}\text { Active phase begins at SNIC } \\
\text { Active phase ends at SNIC }\end{array}$ & Plateau/no undershoot \\
& $\begin{array}{l}\text { Fast subsystem monostable } \\
\text { 2 Slow variables necessary }\end{array}$ & Cannot be reset \\
& $\begin{array}{l}\text { Active phase begins at UH } \\
\text { III }\end{array}$ & Undershoot/no plateau \\
& Active phase ends at SNP & Spike frequency indeterminate \\
& Fast subsystem bistable & Can be reset \\
& 1 Slow variable sufficient & Undershoot/no plateau \\
\hline
\end{tabular}


falls below a voltage plateau, corresponding to the voltage of a fast subsystem saddle point. That is, there is no spike undershoot. In addition, this subsystem is bistable so that the oscillation may be reset by a brief stimulus (i.e. perturbed from a passive to an active state or vice versa).

Type II bursting (Fig. 1B) is characterized by a spike frequency which is low at the beginning, high in the middle, and low again near the end of the active
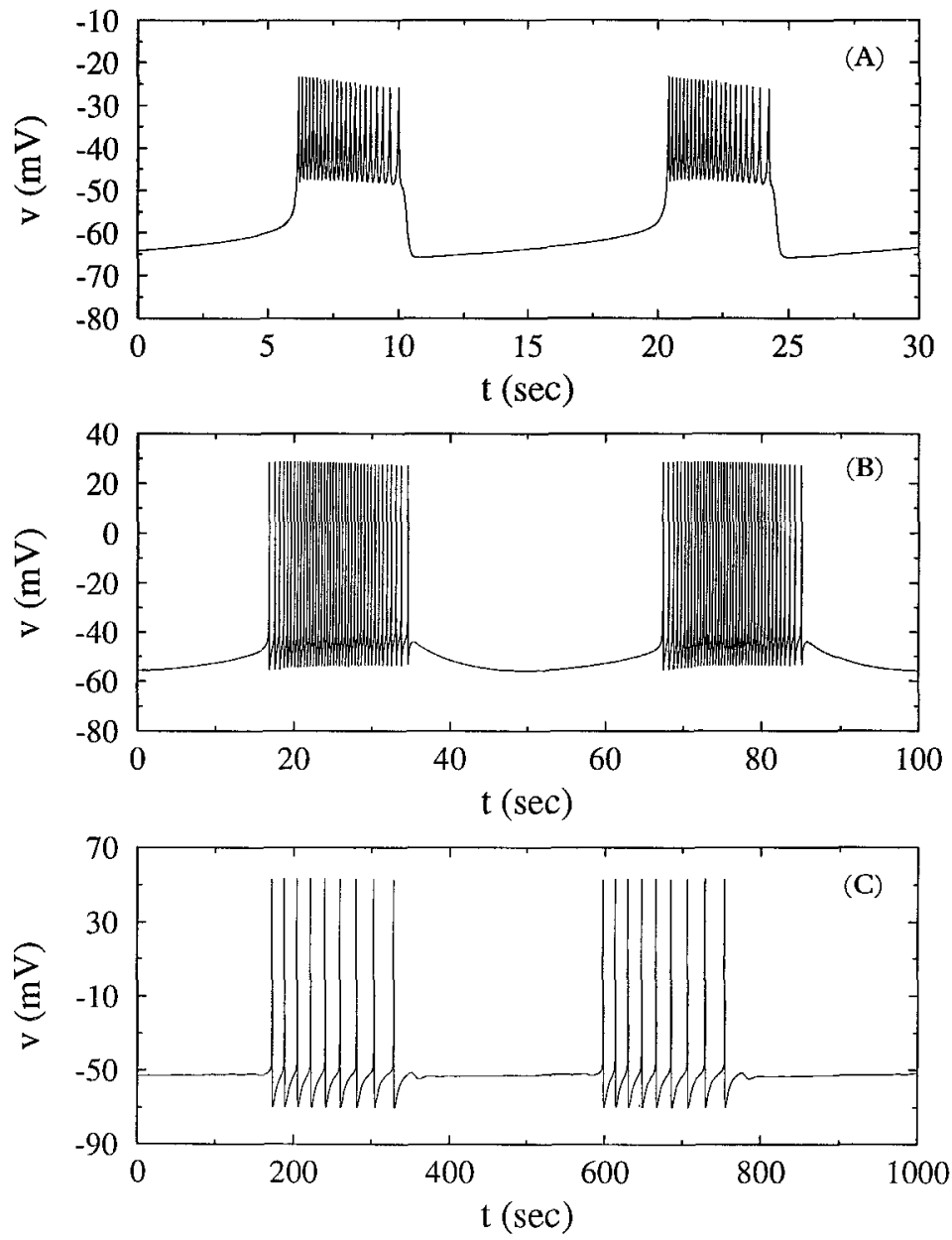

Figure 1. (A) Type I bursting generated by the Sherman-Rinzel model of bursting in pancreatic $\beta$-cells (Sherman and Rinzel, 1992). (B) Type II parabolic bursting generated by the Rinzel-Lee model of bursting in neuron $R_{15}$ of the abdominal ganglion of Aplysia (Rinzel and Lee, 1987). (C) Type III bursting generated by the Av-Ron-Parnas-Segel model of bursting in cardiac ganglion cells of the lobster (Av-Ron et al., 1993). Integration of the systems of ordinary differential equations has been carried out here and throughout the paper using a Gear method (Gear, 1967) as implemented in the LSODE package (Hindmarsh, 1974). 
phase, prompting the name "parabolic bursting". This behavior is due to passage of the bursting trajectory through a SNIC (saddle node on an invariant circle) bifurcation both at the beginning and at the end of the active phase. Spikes undershoot, some falling below the minimum voltage of the silent phase. The fast subsystem is not bistable, so the system cannot be reset by brief stimuli.

Type III bursting (Fig. 1C) has an indeterminate spike frequency profile since no passage is made through a homoclinic bifurcation. This oscillation is characterized by large undershooting spikes with no voltage plateau. Brief stimuli may reset the oscillation due to fast subsystem bistability.

Other bursting oscillations have appeared in the literature which do not, by their appearance, fall neatly into any of these classes. The first example, Fig. 2A, was generated by the model of Pernarowski (1994) and displays a monotonic spike frequency profile like Fig. 1A, but spike undershoot like Fig. 1B and C. This ambiguity is reflected in Pernarowski's reference to it as "nearly" parabolic (Pernarowski, 1994, Fig. 1D). We show that this exemplifies a topologically distinct class of bursting. In common with the oscillation of Fig. 1A, a slow variable makes a single passage through a saddle-loop bifurcation, so we denote this as type Ib and the former as type Ia bursting.

The second example, Fig. 2B, was generated by the $\beta$-cell model of Smolen and Keizer (1992), and, like Fig. 2A, exhibits a monotonic spike frequency profile and spike undershoot. However, we show that this is not another example of type Ib bursting, rather, it is type Ia bursting where the spike undershoot is a result of the increased dimensionality of the fast subsystem.

In the present paper we describe in detail the features defining each topological class of bursting mentioned above and a new one, type IV. In this respect we elaborate on and extend the classification scheme of Rinzel (1987), who described types Ia, II, and III.

Bursting oscillations of all topological classes are generated with the Chay-Cook model (Chay and Cook, 1988), described in section 2. We use a one-parameter bifurcation diagram to describe the structure of the fast subsystem underlying each class of bursting. We locate each one-parameter bifurcation diagram as a horizontal cut through a two-parameter bifurcation diagram. In section 3 we begin the description of this two-parameter bifurcation diagram. We show how a type Ia oscillation can be transformed into a type Ib oscillation by lowering the horizontal cut so that it crosses over a sequence of points representing codimension-two homoclinic (saddle-nodeloop) bifurcations.

In sections 4, 5 and 6 we analyse oscillations of the types shown in Figs 1B, $1 \mathrm{C}$ and $2 \mathrm{~B}$. We complete our description of the two-parameter bifurcation diagram, again representing each type of bursting by a horizontal cut through the diagram. In section 7 we discuss the implications for biological modeling of bursting systems, where information is often limited to the time course of a 
single observable. In such cases, our analysis reveals that phenomenological features are typically insufficient to reliably classify the oscillation.

2. The Chay-Cook Model. The Chay-Cook model (Chay and Cook, 1988) was developed to simulate the bursting electrical behavior of a pancreatic $\beta$-cell. We employ it here for its simplicity and versatility, and for its biophysical character. The model consists of a fast inward or excitatory current $\left(I_{\mathrm{I}}\right)$ carried by $\mathrm{Ca}^{2+}$, a second inward current $\left(I_{\mathrm{S}}\right)$ carried by $\mathrm{Ca}^{2+}$ which may be fast or slow, an outward or inhibitory $\mathrm{K}^{+}$current $\left(I_{\mathrm{K}}\right)$ of the delayed rectifier type, and a passive leakage current $\left(I_{\mathrm{L}}\right)$ :
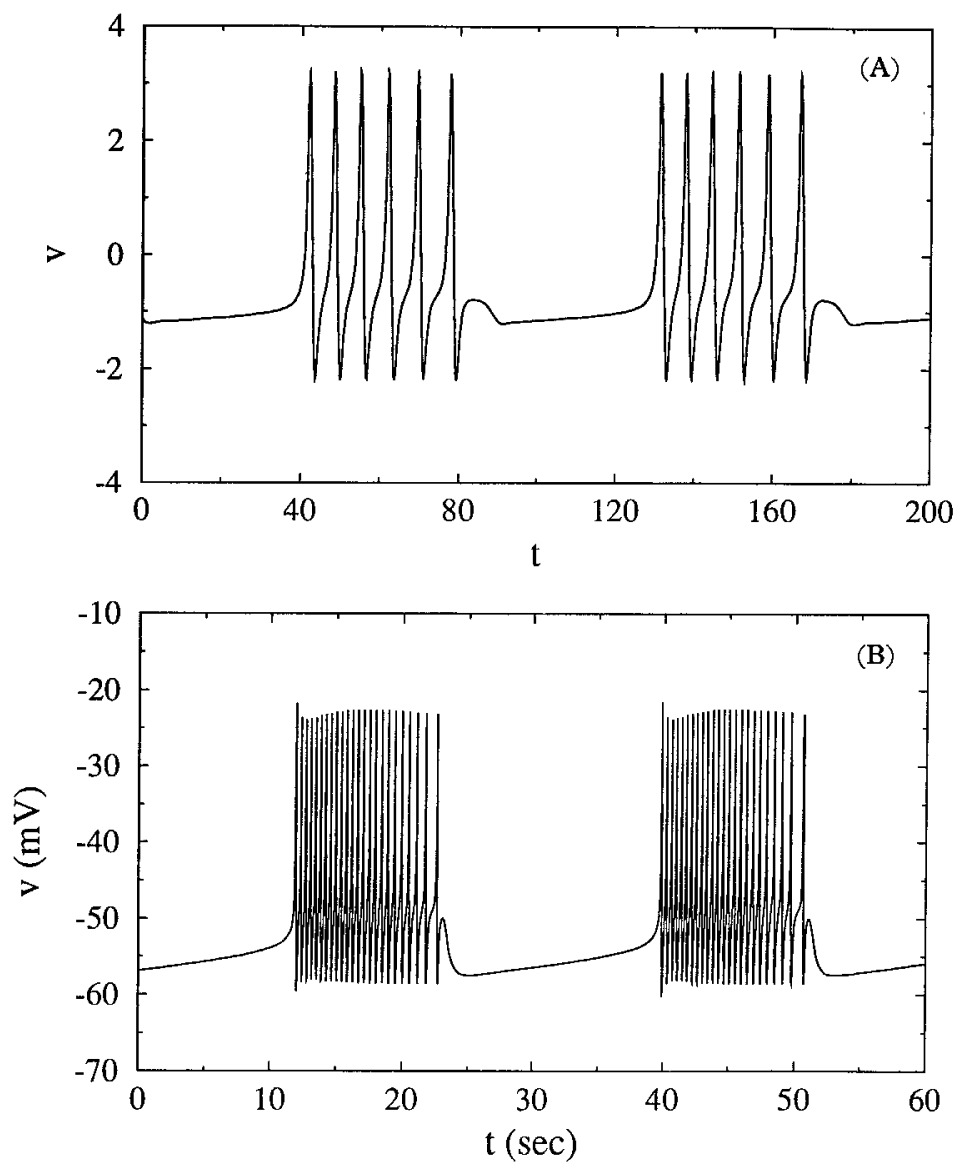

Figure 2. (A) Type Ib bursting generated by the Pernarowski model (Pernarowski, 1994). (B) Type Ia bursting generated by the Smolen-Keizer model of $\beta$-cell bursting (Smolen and Keizer, 1992). Parameters as in their Fig. 6, but with $\lambda=0.84$, $R=0.65$. 


$$
\begin{gathered}
\frac{\mathrm{d} v}{\mathrm{~d} t}=-\left[I_{\mathrm{Ca}}(v, s)+I_{\mathrm{K}}(v, n)+I_{\mathrm{L}}(v)\right] / C_{\mathrm{m}} \\
\frac{\mathrm{d} n}{\mathrm{~d} t}=\lambda\left(n_{\infty}(v)-n\right) / \tau_{\mathrm{n}}(v) \\
\frac{\mathrm{d} s}{\mathrm{~d} t}=\left(s_{\infty}(v, c)-s\right) / \tau_{\mathrm{s}}(v, c) \\
\frac{\mathrm{d} c}{\mathrm{~d} t}=f\left[-\alpha I_{\mathrm{Ca}}(v, s)-k_{\mathrm{c}} c\right] .
\end{gathered}
$$

Here $v$ is the transmembrane potential, $c$ is the intracellular free $\mathrm{Ca}^{2+}$ concentration, $n$ and $s$ are activation variables and the ionic currents are given by

$$
\begin{gathered}
I_{\mathrm{I}}(v)=\bar{g}_{\mathrm{I}} m_{\infty}(v)\left(v-V_{\mathrm{Ca}}\right) \\
I_{\mathrm{S}}(v, s)=\bar{g}_{\mathrm{S}} s\left(v-V_{\mathrm{Ca}}\right) \\
I_{\mathrm{K}}(v, n)=\bar{g}_{\mathrm{K}} n\left(v-V_{\mathrm{K}}\right) \\
I_{\mathrm{L}}(v)=\bar{g}_{\mathrm{L}}\left(v-V_{\mathrm{L}}\right) \\
I_{\mathrm{Ca}}(v, s)=I_{\mathrm{I}}(v)+I_{\mathrm{S}}(v, s) .
\end{gathered}
$$

The steady-state functions and activation times are

$$
\begin{gathered}
x_{\infty}(v)=\frac{1}{1+\exp \left[\left(V_{x}-v\right) / S_{x}\right]},(x=m, n) \\
s_{\infty}(v, c)=\frac{1}{1+\exp (2 A(v, c))} \\
\tau_{\mathrm{n}}(v)=\frac{\bar{\tau}_{\mathrm{n}}}{1+\exp \left[\left(v-V_{\mathrm{n}}\right) / S_{\mathrm{n}}\right]} \\
\tau_{\mathrm{s}}(v, c)=\frac{\bar{\tau}_{\mathrm{s}}}{2 \cosh (A(v, c))},
\end{gathered}
$$


where

$$
A(v, c)=\frac{V_{\mathrm{s}}+S_{\mathrm{s}} \log (\tilde{c})-v}{2 S_{\mathrm{s}}}
$$

and $\tilde{c}=c /(1 \mu \mathrm{M})$. The parameters that remain unchanged in our computations are $\bar{g}_{\mathrm{I}}=250 \mathrm{pS}, \bar{g}_{\mathrm{S}}=10 \mathrm{pS}, \bar{g}_{\mathrm{K}}=1300 \mathrm{pS}, \bar{g}_{\mathrm{L}}=50 \mathrm{pS}, V_{\mathrm{Ca}}=100 \mathrm{mV}, V_{\mathrm{K}}=$ $-80 \mathrm{mV}, V_{\mathrm{L}}=-60 \mathrm{mV}, C_{\mathrm{m}}=4524 \mathrm{fF}, \bar{\tau}_{\mathrm{n}}=9.09 \mathrm{msec}, V_{\mathrm{m}}=-22 \mathrm{mV}, V_{\mathrm{n}}=$ $-9 \mathrm{mV}, \quad V_{\mathrm{s}}=-22 \mathrm{mV}, \quad S_{\mathrm{m}}=7.5 \mathrm{mV}, \quad S_{\mathrm{n}}=10 \mathrm{mV}$ and $\alpha=5.727 \times$ $10^{-6} \mathrm{fA}^{-1} \mu \mathrm{M} \mathrm{msec}{ }^{-1}$.

The effective time constant for the potassium activation variable $n$ is $\tau_{\mathbf{n}} / \lambda$; $\lambda$ is the primary bifurcation parameter in our analysis. We also vary $\bar{\tau}_{\mathrm{s}}$ to modify the role played by the $\mathrm{Ca}^{2+}$ current activation variable $s$. When $\bar{\tau}_{\mathrm{s}}$ is small ( 1 sec or less) the system can burst given bistability of the fast subsystem (1-3) and appropriate modulation by the slow variable $c$. When $\bar{\tau}_{\mathrm{s}}$ is large $(10 \mathrm{sec}) \mathrm{s}$ is a slow variable and bursting can be generated as the two-dimensional slow subsystem (3-4) interacts with the fast subsystem to generate a slow oscillation, periodically moving the fast dynamics between monostable quiescent and oscillatory states. For given values of $\lambda$ and $\bar{\tau}_{\mathrm{s}}$ we modify slow subsystem parameters $\left(f, k_{\mathrm{c}}\right.$ and, when $s$ is slow, $S_{\mathrm{s}}$ ) to values conducive to bursting. The latter changes do not affect the fast subsystem bifurcation structures, on which the bursting classification is based.

3. Transition from Type Ia to Type Ib Bursting. In this section we describe the transition from type Ia to type Ib bursting, and begin the description of a twoparameter bifurcation diagram which provides a framework for the classification of bursting oscillations. This description is completed in section 4, where we discuss type III and IV bursting.

Most models of bursting involving a single slow variable yield type Ia oscillations, with small spikes riding on a voltage plateau. It may appear that large undershooting spikes are incompatible with bursting driven by a single slow variable. We show that they are compatible, by demonstrating that type Ia bursting can be transformed into large-spike type Ib bursting.

We analyse first a three-dimensional version of the Chay-Cook model obtained by setting $s=s_{\infty}(v, c)$, therefore confining the fast subsystem dynamics to the $(v, n)$-plane. We refer to this as Chay-Cook- $(2,1)$ (two fast variables and one slow). With $\lambda=0.95$ a type Ia oscillation is generated (Fig. 3A), which is dissected in Fig. 4A. To construct a fast subsystem bifurcation diagram, $c$ is treated as a bifurcation parameter and the $v$-values of the equilibrium states are plotted, generating the Z-shaped slow manifold (SM). The spikes of the bursting oscillation are periodic solutions of the fast subsystem, for which the maximum and minimum $v$ are plotted.

One can think of $c$ as a slowly-varying parameter, leading the full-system 
trajectory along solution branches of the fast subsystem. At the beginning of the silent phase, the phase point lies on the bottom branch of the slow manifold and thus below the slow nullcline, $\mathrm{d} c / \mathrm{d} t=0$. Since $\mathrm{d} c / \mathrm{d} t<0$, the phase point moves leftward until the manifold switches back via a saddle-node bifurcation and the phase point is attracted to the periodic branch, initiating the active phase of bursting. The phase point now lies above the slow nullcline, so it moves rightward as it oscillates. The active phase is terminated when a spike reaches the middle branch of the slow manifold, returning the phase point to the lower branch to restart the silent phase.

For any $c$ between the left knee of the slow manifold and the termination of the periodic branch, the $(v, n)$-phase plane contains a stable stationary point, a saddle point, and an unstable stationary point which is enclosed by a tear-
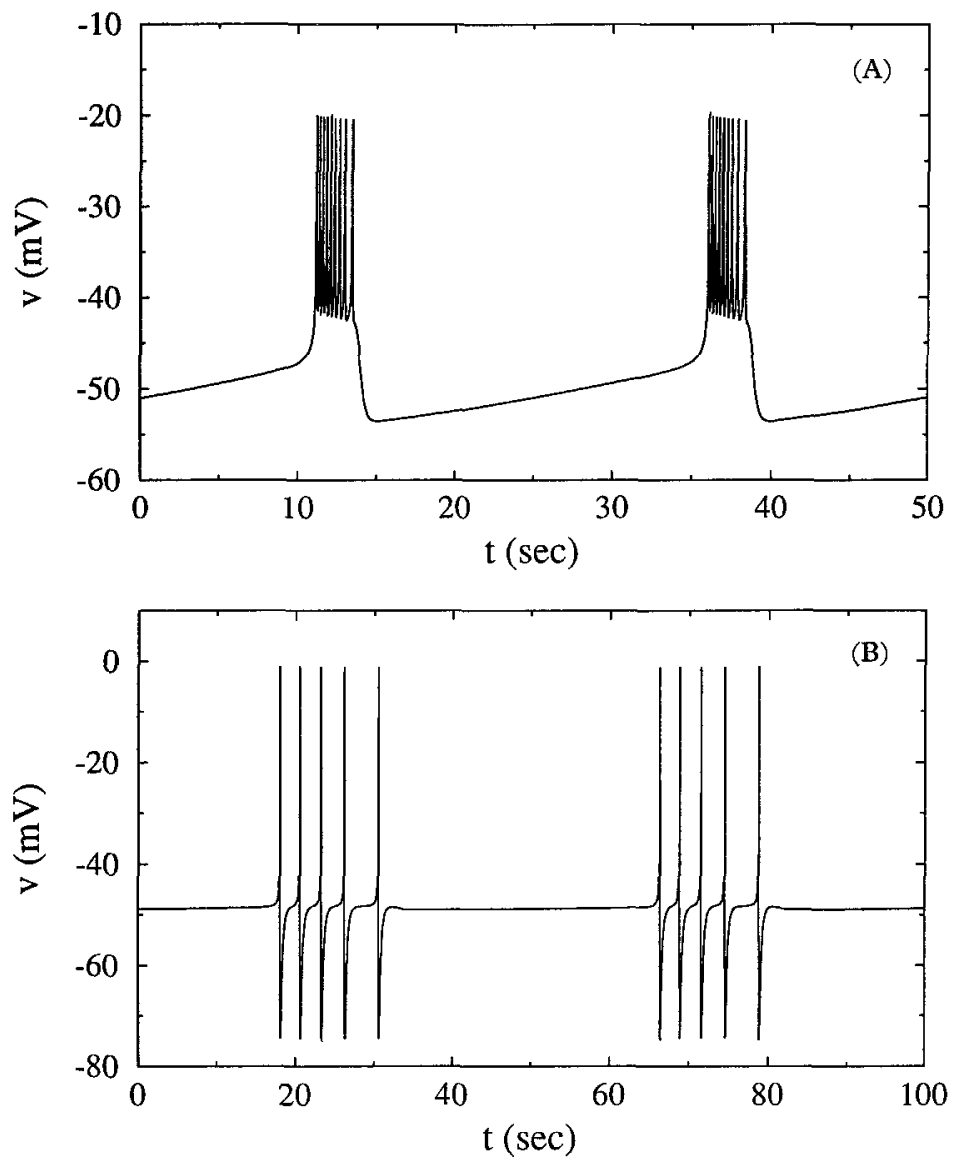

Figure 3. (A) Type Ia bursting generated by Chay-Cook- $(2,1)$ with $\lambda=0.95$, $f=0.002$. (B) Type Ib bursting with $\lambda=0.17, f=5 \times 10^{-5}$. In both cases $k_{\mathrm{c}}=0.027 \mathrm{~m} \mathrm{sec}^{-1}$ and $S_{\mathrm{s}}=10 \mathrm{mV}$. 
shaped stable limit cycle. The stationary points are the three intersections of the fast subsystem nullclines (Fig. 5). As $c$ is increased the limit cycle grows and connects with the saddle point when both $n$ and $v$ are at their minimum values over the oscillation, thus forming an infinite-period saddle-loop orbit. As $c$ is
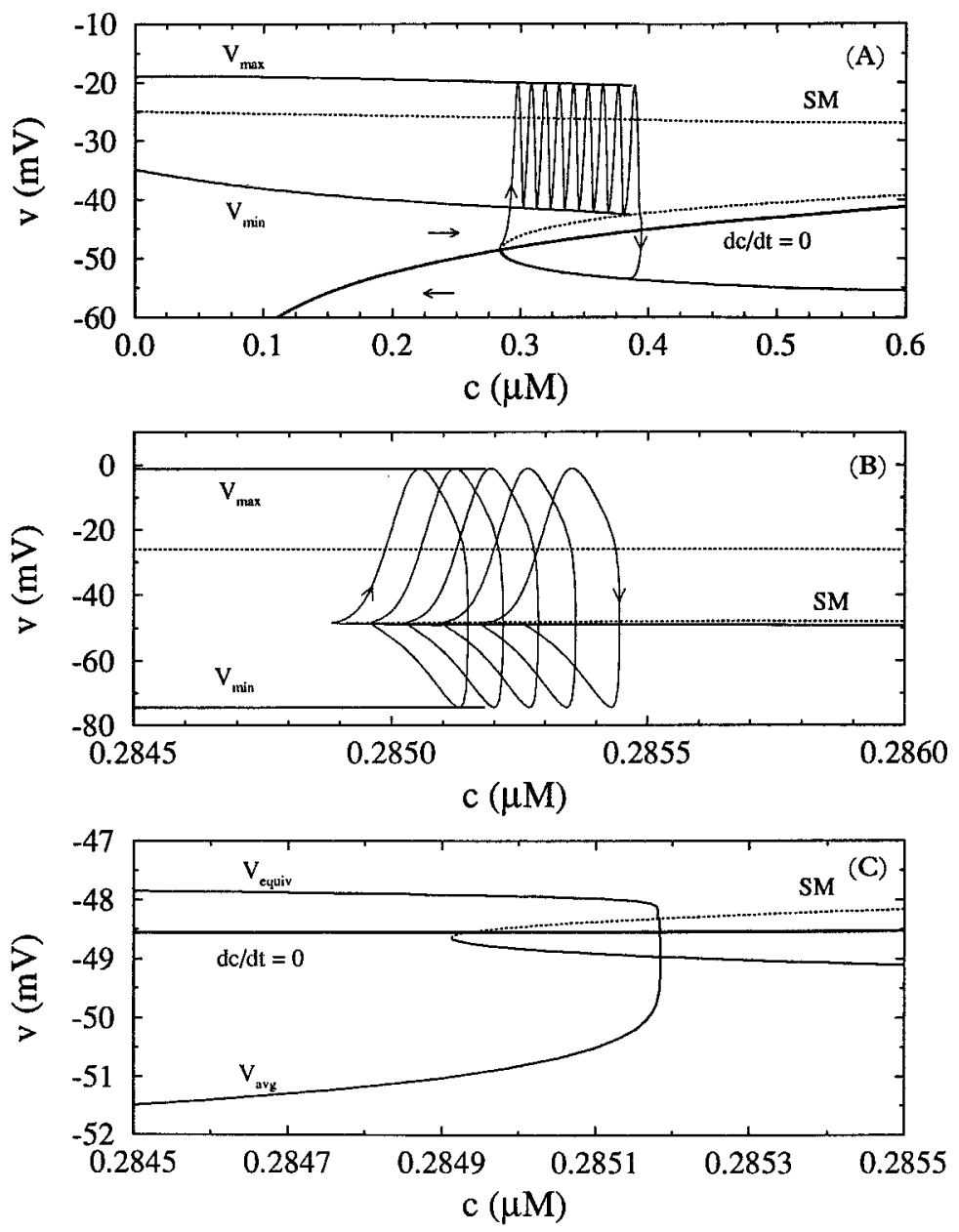

Figure 4. Dissection of type Ia and Ib bursting generated by Chay-Cook- $(2,1)$ with $k_{\mathrm{c}}=0.027 \mathrm{~m} \mathrm{sec}^{-1}, S_{\mathrm{s}}=10 \mathrm{mV}$, and (A) $\lambda=0.95, f=0.002$, (B) $\lambda=0.17, f=$ $5 \times 10^{-5}$. The fast subsystem slow manifold (SM) is the Z-shaped curve consisting of stable (solid) and unstable (dotted) branches. The periodic branch is represented by the minimum and maximum $v$ of the periodic solution. The projection of the bursting orbit is superimposed. The slow nullcline, $\mathrm{d} c / \mathrm{d} t=0$, is included in (A) with accompanying arrows to indicate the direction of flow on either side. Note the much smaller range of variation of $c$ in (B). In (C) the left knee of the slow manifold from (B) is magnified. Also included is the slow nullcline and the $v_{\text {avg }}$ and $v_{\text {equiv }}$ curves. Bifurcation diagrams are constructed here and throughout the paper using the computer program AUTO (Doedel, 1981). 
increased further the connection breaks and the limit cycle is annihilated. Thus, the spike voltage never falls below the saddle, and there is no spike undershoot. In addition, because the periodic branch terminates with an infinite-period orbit, the frequency of spiking decays monotonically as the end of the active phase of bursting is approached (this does not imply that overall frequency profile is monotonic, but it usually is).

We contrast this oscillation with that of Fig. 3B, which also exhibits a monotonic spike frequency profile, but displays large undershooting spikes. This oscillation is obtained by decreasing the fast subsystem parameter $\lambda$ and the slow subsystem parameter $f$. The latter is a multiplicative constant affecting only the rate of change of $c$. The former also has no effect on the slow manifold or nullcline, but affects the periodic branch, which now encloses the lower and middle branches of the slow manifold as well as the upper branch (Fig. 4B).

How can such a transition be made, given that the periodic branch cannot pass through the slow manifold without trajectories crossing in the $(v, n)$-plane? As $\lambda$ is decreased, the saddle-loop bifurcation point in Fig. 4A moves leftward along the middle branch of the manifold until the knee is reached. At this point, a saddle-node-loop bifurcation (SNL), the minimum $v$ moves vertically downward, with the average spike $v$ remaining at the knee. Eventually a second SNL occurs and the minimum $v$ of the homoclinic orbit switches back underneath the bottom branch of the manifold (like the HM curve in Fig. 9B), while the average $v$ moves back along the middle branch. While the homoclinic is at the knee the fast subsystem is monostable, so the full

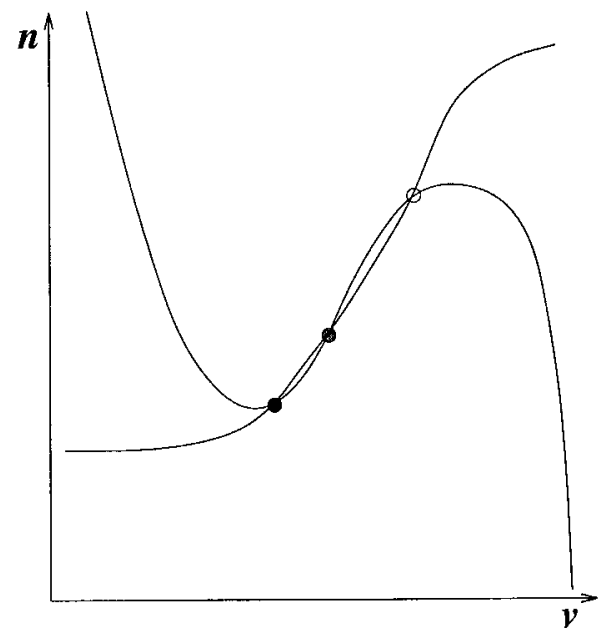

Figure 5. Schematic illustration of fast subsystem nullclines. The sigmoidal and cubic-like curves represent the $n$ and $v$ nullclines of Chay-Cook- $(2,1)$. Black and white circles represent stable and unstable stationary solutions, while the gray circle represents a saddle solution, of the fast subsystem. 
system can exist in either a quiescent or continuous spiking state, but cannot burst with a single slow variable (but see section 6, where type II bursting is discussed).

Once the second SNL has been encountered the fast subsystem is again bistable and capable of bursting. However, the spikes are now large, enclosing all three branches of the slow manifold (Fig. 4B). This bursting, here denoted type $\mathrm{Ib}$, is topologically distinct from the small-spike bursting of Fig. 4A, as evident from the schematic representation of the phase portraits in the $(v, n)$-plane, for some $c$ just to the right of the left knee of the slow manifold (Fig. 6). When $\lambda=0.95$, the phase plane contains a stable stationary point, a saddle, and an unstable stationary point surrounded by a stable limit cycle (Fig. 6A). As $\lambda$ is decreased the limit cycle grows until it forms a saddle-loop orbit (Fig. 6B). The SL breaks as $\lambda$ is further decreased, leaving the stable point as the global attractor (Fig. $6 \mathrm{C}$ ). With smaller $\lambda$ one branch of the saddle's unstable manifold coalesces with a branch of its stable manifold, forming a second SL (Fig. 6D). This orbit disconnects from the saddle when $\lambda$ is made smaller and persists as a stable limit cycle encircling all three equilibria (Fig. 6E), which is the case when $\lambda=0.17$ (Fig. 4B).

Figure 7 shows a two-parameter bifurcation diagram with parameters $\sigma$ and $\mu$, here representing $c$ and $\lambda$, which we will use to represent classes of bursting. The horizontal parameter $\sigma$ is the slow variable appearing as a parameter in the fast subsystem. The vertical parameter $\mu$ is a parameter in the fast subsystem which remains fixed for the full bursting system. Every one-parameter bifurcation diagram of the fast subsystem is represented as a horizontal cut through the two-parameter bifurcation diagram. This figure is schematic, representing curves constructed with Chay-Cook- $(2,1)$, as well as other versions of the Chay-Cook model (employed in later sections), using the computer program AUTO (Doedel, 1981).

The stable saddle-loop orbits (SSL) of Fig. 6B and D illustrate the phase portraits along the two-parameter bifurcation curves $\mathbf{B}$ and $\mathbf{D}$ in Fig. 7. (We refer to a bifurcation as either stable or unstable according to the stability of the limit cycle it produces.) The transition from Fig. 6A (small spikes) to Fig. 6E (large spikes) starts in region $\mathbf{A}$ and terminates in region $\mathbf{E}$ after passing through region $\mathbf{C}$ and the two SSL curves $\mathbf{B}$ and $\mathbf{D}$.

The left and right knees or saddle-node bifurcations (SN) of the slow manifold appear as vertical lines in Fig. 7. Curves $\mathbf{B}$ and $\mathbf{D}$ are tangent to the left SN curve at a pair of codimension-two stable saddle-node-loop (SSNL) bifurcation points (Schecter, 1987). Between these points on the SN curve the homoclinic orbit deforms continuously, forming a line segment of stable codimension-one SNIC bifurcations (Guckenheimer and Holmes, 1983, p. 149; Ermentrout and Kopell, 1986). Curve B connects tangentially with the right SN curve at a codimension-two Takens-Bogdanov (TB) bifurcation 
point (Guckenheimer and Holmes, 1983, p. 364) where both the trace and the determinant are zero. From such points a curve of Hopf bifurcations also emerges. These Hopf points are supercritical, giving rise to oscillations which terminate on curve B. The curve of supercritical Hopf (SH) bifurcations extends past the left $\mathrm{SN}$ curve.

Type Ia bursting is represented in Fig. 7 as a horizontal line segment between the left SN curve (where the silent phase terminates) and the SL curve B (where the active phase terminates). Between these two endpoints the segment lies in region $\mathbf{A}$ and thus the active phase has non-undershooting spikes. Type $\mathrm{Ib}$ bursting also extends between the left SN curve and an SL curve, but lies in region $\mathbf{E}$ where the spiking oscillation surrounds all three equilibria, giving rise to bursting with spike undershoot.

Types Ia and Ib both have appropriate fast dynamics, including bistability, to burst with a single slow variable. In addition, there are constraints on the

$\mathbf{A}$

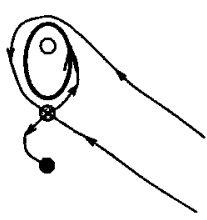

D

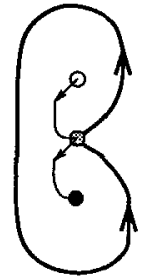

G

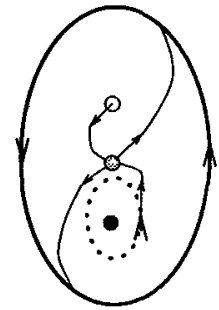

B

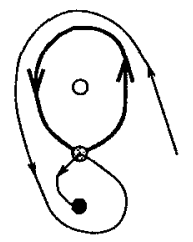

$\mathbf{E}$

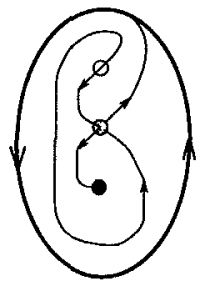

H

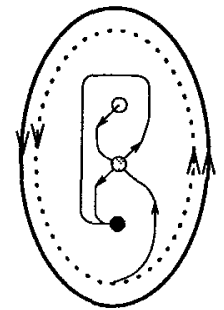

C

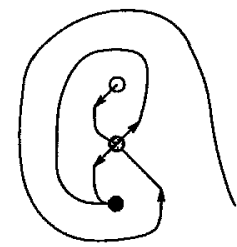

$\mathbf{F}$

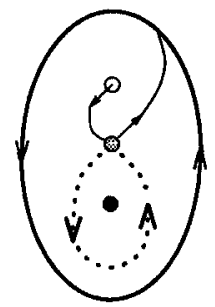

I

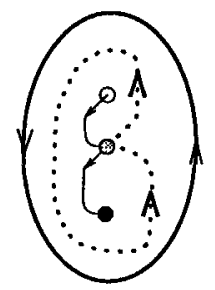

Figure 6. Schematic fast subsystem phase plane diagrams illustrate topological, but not metric, features of orbits accurately. Circles represent equilibrium points, black being stable, white unstable, and gray a saddle. Thin directed curves represent branches of the saddle's stable and unstable manifolds. Thick directed curves represent stable limit cycles. Dotted directed curves represent unstable limit cycles. Each frame is identified with a letter corresponding to a region or a curve in Fig. 7. 
slow dynamics that can be derived formally by averaging the slow dynamics over the spikes. For the case of Chay-Cook $(2,1)$, the averaged slow equation is

$$
\frac{\mathrm{d} c}{\mathrm{~d} t}=f\left(-\frac{\alpha}{T(c)} \int_{0}^{T(c)} I_{\mathrm{Ca}}(v(t, c), c) \mathrm{d} t-k_{\mathrm{c}} c\right)
$$

where $T(c)$ is the period of the fast subsystem limit cycle $v(t, c)$ corresponding to each fixed value of $c$. For bursting with given fast subsystem parameters, $k_{\mathrm{c}}$ must be chosen so that $c$ decreases during the silent phase and increases during the active phase. For the silent-phase condition, the $c$ nullcline must be above the knee since $v$ is at quasi-steady state. For the active-phase, it is sufficient for the $c$ nullcline to be below the minimum $v$ curve of the periodic branch (Fig. 4A), but not necessary, as seen in Fig. 4B where $v$ goes below the bottom

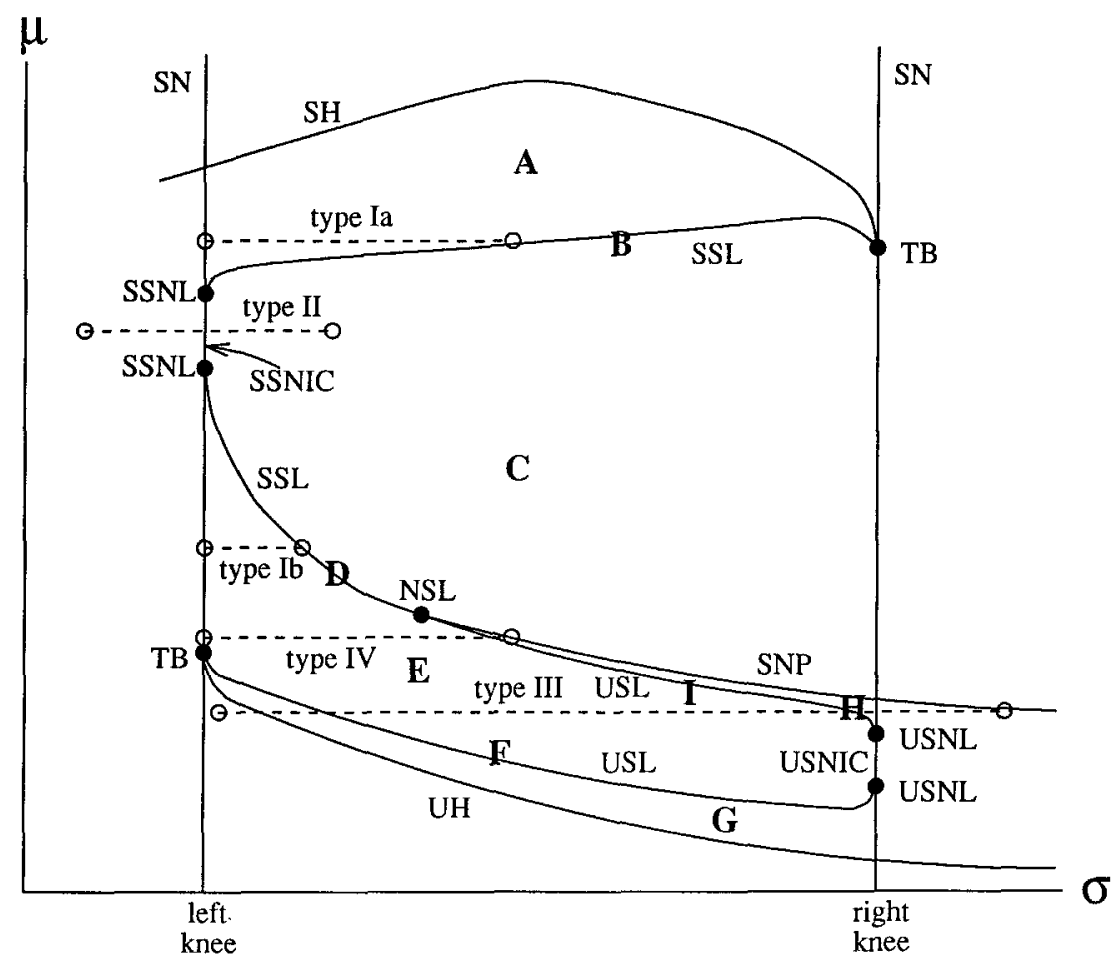

Figure 7. Schematic fast subsystem two-parameter bifurcation diagram. $\sigma$ is a variable of the slow subsystem $(c$ or $1-s)$ and $\mu$ is a fast subsystem parameter $(\lambda)$. Curves represent codimension-one bifurcations. Filled circles represent codimension-two bifurcations. The five classes of bursting described in the text are represented by dashed horizontal lines. A, C, E, G and $\mathbf{H}$ label regions, while B, D, F and I label curves. 
branch of the $Z$-curve. A graphical way to determine whether the system will burst is to define an equivalent voltage, $v_{\text {equiv }}$, implicitly by

$$
I_{\mathrm{Ca}}\left(v_{\text {equiv }}(c), c\right)=\frac{1}{T(c)} \int_{0}^{T(c)} I_{\mathrm{Ca}}(v(t, c), c) \mathrm{d} t
$$

and solve numerically for $v_{\text {equiv }}(c)$ (Fig. 4C). Then (15) becomes

$$
\frac{\mathrm{d} c}{\mathrm{~d} t}=f\left(-\alpha I_{\mathrm{Ca}}\left(v_{\text {equiv }}(c), c\right)-k_{\mathrm{c}} c\right) .
$$

This is positive if $\left(c, v_{\text {equiv }}(c)\right)$ lies above the $c$ nullcline. Thus, the system bursts if the $c$ nullcline lies between the knee and the $v_{\text {equiv }}$ curve.

Note that the curve of average voltage,

$$
v_{\mathrm{avg}}(c)=\frac{1}{T(c)} \int_{0}^{T(c)} v(t, c) \mathrm{d} t
$$

lies below the $c$ nullcline. Although the spikes spend much time at low voltages, $-\alpha I_{\mathrm{Ca}}$ weights the high voltages more, and the net effect of each spike is to increase $c$.

Both the $v_{\text {avg }}$ and $v_{\text {equiv }}$ curves terminate on the middle branch of the slow manifold in a saddle-loop. The other termination of those curves is at a Hopf bifurcation, off the page to the left.

4. Type III and Type IV Bursting. In both of the bursting oscillations discussed thus far the active phase of bursting has terminated via a saddle-loop bifurcation, with the consequent decrease of spike frequency as this point is approached. In this section we again use Chay-Cook- $(2,1)$ to discuss two additional classes of bursting, which do not involve homoclinic termination of the active phase. These oscillations are obtained by further decreasing $\lambda$ in (2).

As $\lambda \rightarrow 0$, the relaxation limit, the lowermost equilibrium point of the fast subsystem (1-2) destablizes via Hopf bifurcation if and only if it lies on the middle branch of the $v$ nullcline (Fig. 5). For $c$ sufficiently close to the left knee of the slow manifold this will be the case, since the $n$ nullcline has positive slope. Thus, a portion of the lower branch of the slow manifold destablizes as $\lambda \rightarrow 0$ (Fig. 8B).

The branch of unstable oscillations born at the Hopf bifurcation, which is subcritical, terminates in a saddle loop. As $\lambda$ is decreased, the saddle-loop and the Hopf point move rightward in the $(c, v)$ diagram, forming curves $\mathbf{F}$ and $\mathrm{UH}$ in Fig. 7. These curves emerge from a TB bifurcation point at the left knee of the slow manifold.

The stability of the infinite-period orbit that emerges at a saddle-loop 
bifurcation is determined by the trace of the linearization about the saddle point (Guckenheimer, 1986). The trace is negative on $\mathbf{D}$, so a stable orbit emerges. However, the trace switches sign at a codimension-two neutral saddle-loop bifurcation (NSL), beyond which, on I, the emergent saddle-loop orbit is unstable (Fig. 6I). Therefore, emerging from the NSL is a saddle node of periodics (SNP) curve. One can locate the NSL bifurcation by finding the point of intersection of the SL curve with the curve of equilibrium points with zero trace (not shown), which runs continuously between the TB points, where it is tangent to the SN curve and becomes a curve of Hopf points.

Finally, the unstable SL curves I and $\mathbf{F}$ terminate at the right $\mathbf{S N}$ via unstable SNL bifurcations joined by an interval of unstable SNIC bifurcations.
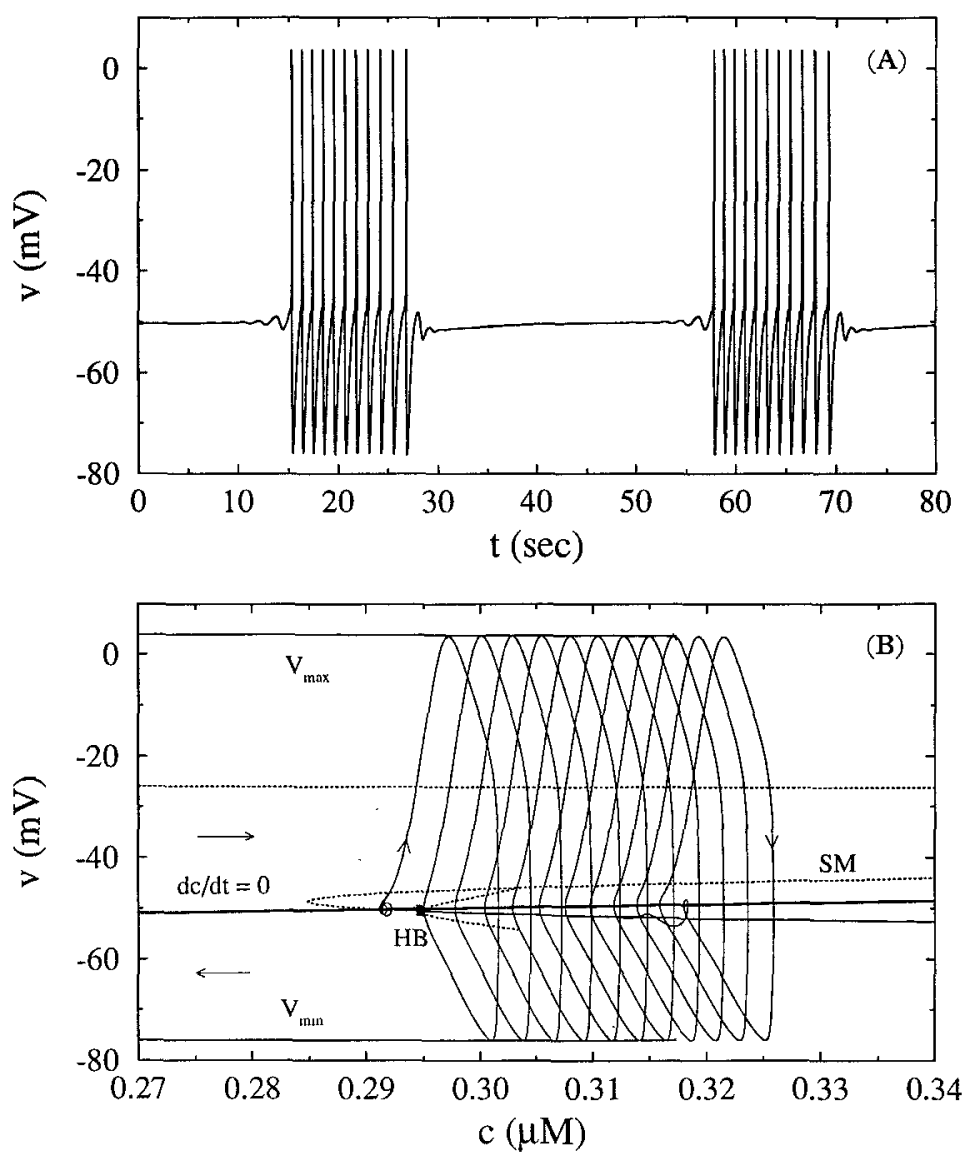

Figure 8. (A) Type III oscillation generated by Chay-Cook- $(2,1)$ with $\lambda=0.1$, $f=0.002, k_{\mathrm{c}}=0.022 \mathrm{~m} \mathrm{sec}^{-1}$, and $S_{\mathrm{s}}=10 \mathrm{mV}$. (B) Dissection of the oscillation. The large amplitude periodic branch (solid) is stable while the small amplitude branch (dotted), emanating from a subcritical Hopf bifurcation (HB), is unstable. 
In type IV bursting, represented in Fig. 7, the silent phase is terminated at a knee of the slow manifold while the active phase terminates at a saddle node of periodics. Type III bursting runs between a subcritical Hopf bifurcation and a SNP bifurcation, its representation in Fig. 7 being any horizontal line segment below the TB point connecting the UH and SNP curves. Both oscillations run through regions $(\mathbf{E}, \mathbf{H}$, and $\mathbf{G}, \mathbf{E}, \mathbf{H}$, respectively) which involve bistability between a stationary point and a limit cycle encircling all three stationary points. Hence, only one slow variable is required to generate the oscillations (Fig. 8), which exhibit large undershooting spikes and may be reset by instantaneous perturbations. In addition, since the active phase is neither initiated nor terminated via homoclinic bifurcation, the spike frequency may be monotonic, parabolic, or neither.

In the type III oscillation dissected in Fig. $8 \mathrm{~B}$, the phase lies below the $c$ nullcline on the stable portion of the slow manifold during the silent phase. Since $\mathrm{d} c / \mathrm{d} t<0$ the trajectory moves leftward along the manifold and ramps through the Hopf bifurcation, oscillating with growing amplitude until it reaches the periodic branch. Then, the trajectory moves rightward while oscillating until it reaches the saddle node of periodics. Finally, it returns to the slow manifold with a few damped oscillations. The ramping that is inherent in passage through a Hopf bifurcation (Baer et al., 1989) and that is responsible for post silent-phase small oscillations in type III bursting is represented in Fig. 7 by placing the silent phase termination point to the left of the UH curve. Phenomenologically, type IV differs from type III in that small oscillations occur only before, not after, the silent phase. However, if the Hopf bifurcation in type III is close to the knee, the trajectory may ramp all the way to the knee, and no oscillations will be seen after the silent phase.

5. Undershooting Spikes With Non-planar Fast Subsystems. Although the two-parameter bifurcation diagram (Fig. 7) was motivated by the phase plane diagrams of Fig. 6, its basic structure persists in higher dimensions. That is, bursting oscillations generated by models with more than two fast variables can be classified according to the horizontal line segment in Fig. 7 visited by the bursting orbit. However, some of the phenomenological features associated with different classes of oscillations when the fast subsystem is planar are no longer observed in higher dimensions. In particular, type Ia bursting, which does not display spike undershoot when the fast subsystem is planar, can do so in higher dimensions. This is observed, for example, in the type Ia oscillation generated by the Smolen-Keizer model (Fig. 2B) which has five fast variables.

To illustrate this we use the full Chay-Cook model (1-4), with $\bar{\tau}_{\mathrm{s}}=1 \mathrm{sec}$, referring to this as Chay-Cook- $(3,1)$. This time constant is sufficiently small that $s$ plays the role of an additional fast variable, raising the dimensionality of the fast subsystem to three. When $\lambda=0.6$ the system exhibits type Ia bursting, 
with the silent phase terminating at a saddle node of the slow manifold and the active phase terminating at a saddle-loop bifurcation (Fig. 9A).

Unlike Fig. 4A the bursting trajectory undershoots the middle branch of the slow manifold. As a result, following the last spike a voltage "hump" is present as the trajectory approaches, but does not reach, the spike threshold (Fig. 10).

When $s$, rather than $v$, is plotted against $c$ in the bifurcation diagram, it is clear that the periodic branch terminates when the minimum-s curve intersects the middle branch of the slow manifold (Fig. 9B). Hence, the saddle-loop orbit connects with the saddle point when $s$ is at its minimum over a spike, rather than when $v$ is at its minimum. This is illustrated in Fig. 11, for $c$ fixed at three values close to the homoclinic. There are three clockwise-oriented orbits,
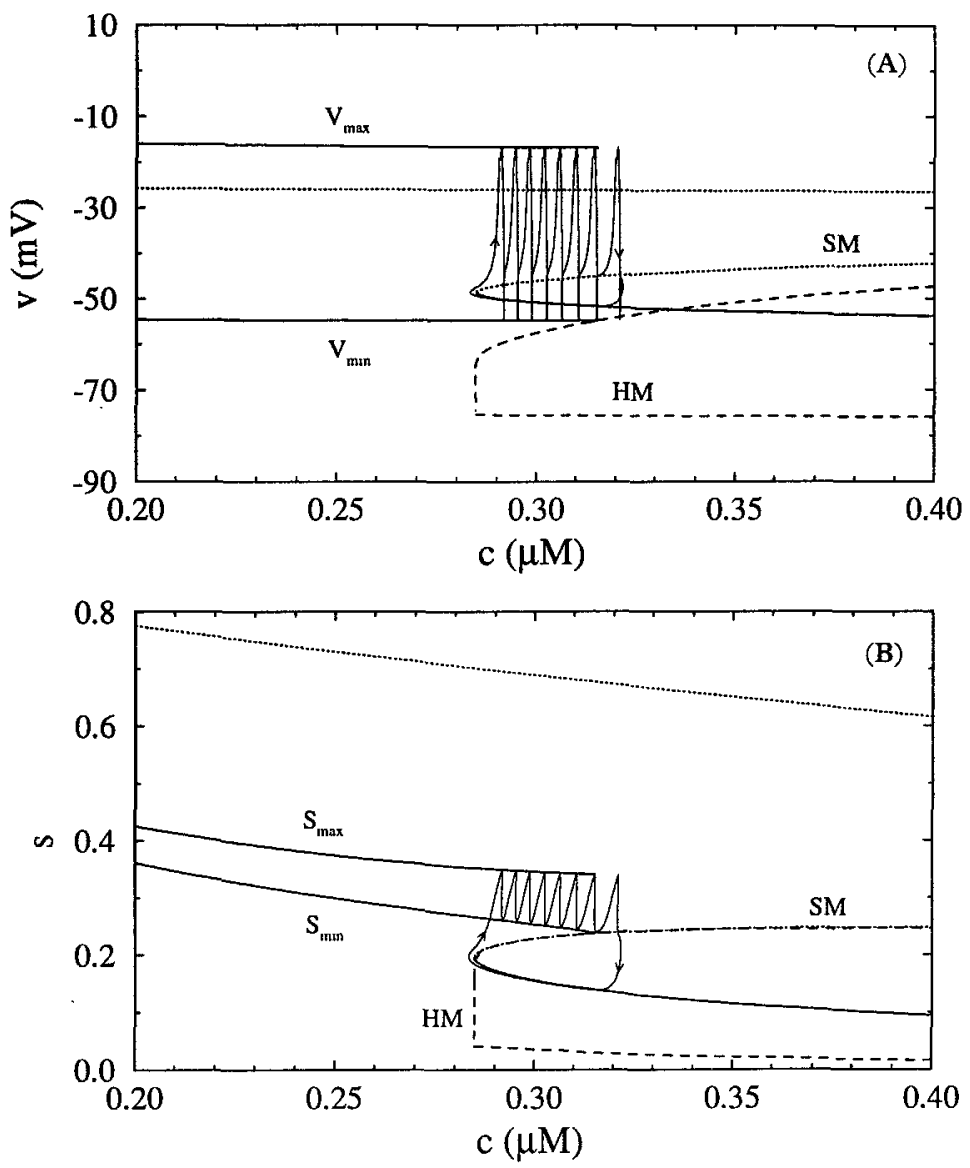

Figure 9. Dissection of type Ia bursting generated by Chay-Cook-(3, 1) with $\bar{\tau}_{\mathrm{s}}=1 \mathrm{sec}, \lambda=0.6, f=5 \times 10^{-4}, k_{\mathrm{c}}=0.027 \mathrm{~m} \mathrm{sec}^{-1}$, and $S_{\mathrm{s}}=10 \mathrm{mV}$. (A) $v$ vs $c$; (B) $s$ vs $c$. Homoclinic curves (HM) showing minimum $v$ and $s$ are traced out for $\lambda \in[0.02$, $0.8]$. 
corresponding to periodic spiking solutions of the fast subsystem. When $c=0.28 \mu \mathrm{M}$ the orbit is far from the saddle point, while by $c=0.316 \mu \mathrm{M}$ it has deformed and made contact. Notice that the minimum voltage of this latter orbit is below the $v$-value of the saddle point and that the saddle connection is made in the ascending phase of the spike where $s$ is at its minimum.

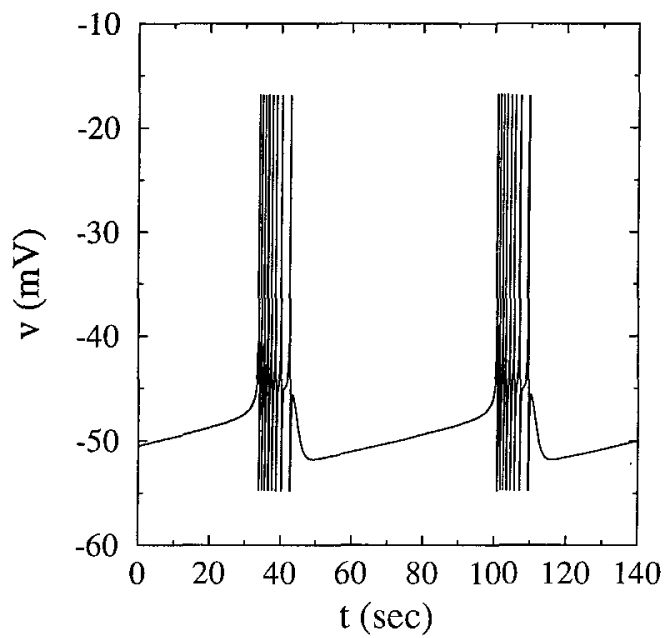

Figure 10. Chay-Cook- $(3,1)$ bursting with $\lambda=0.6, f=5 \times 10^{-4}, k_{\mathrm{c}}=0.027 \mathrm{~m} \mathrm{sec}^{-1}$, $S_{\mathrm{s}}=10 \mathrm{mV}$. Note the voltage hump following the last spike of each burst, indicating the presence of spike undershoot. Similar type Ia oscillations were shown in Fig. 3 of Chay and Cook (1988).

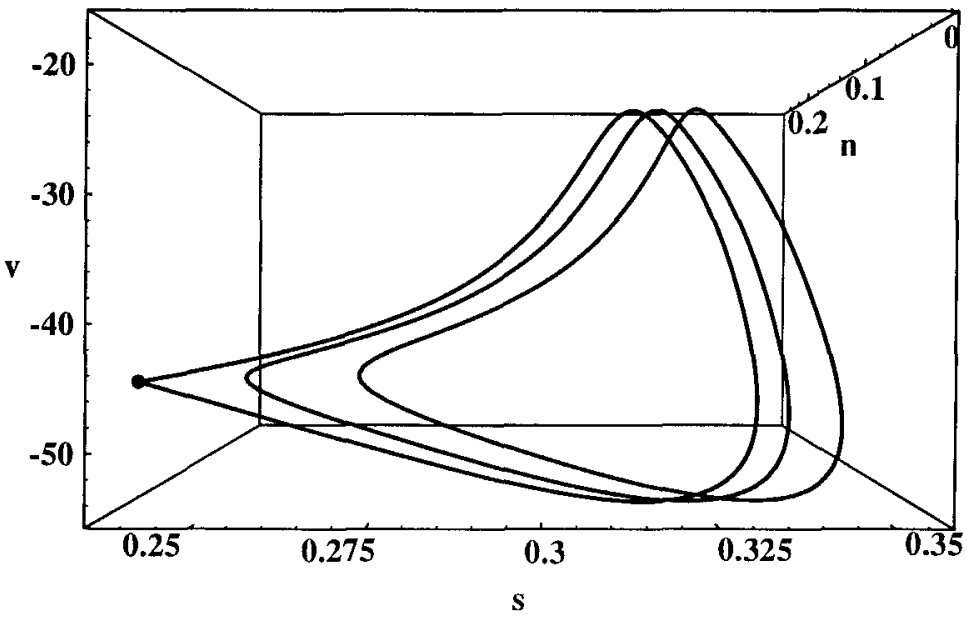

Figure 11. Chay Cook- $(3,1)$ spike orbits for $\lambda=0.6$ and $c=0.28,0.3$, and $0.316 \mu \mathrm{M}$. When $c=0.316 \mu \mathrm{M}$ the orbit connects with the saddle point to form an infinite period saddle-loop. 
As in Chay-Cook- $(2,1)$ the fast subsystem's one-parameter bifurcation diagram goes through two SNL bifurcations as $\lambda$ is decreased. This is illustrated in Fig. 9, where the minimum $v$ (or $s$ ) of the homoclinic orbit is traced out over a range of values of $\lambda$ (curve HM). Here we see again that the saddle-loop orbit undershoots the middle branch of the slow manifold during type Ia bursting. In addition, we see that Chay Cook- $(3,1)$ is capable of type Ib bursting.

6. Type II Bursting. In the scenarios discussed thus far a necessary condition for bursting has been bistability of the fast subsystem. If there are two or more slow variables, however, bistability may not be necessary. In this section we review how bursting can be achieved when the fast subsystem is monostable; our case is similar to that of Rinzel and Lee (1987). When represented in the two-parameter diagram (Fig. 7), this bursting is seen to form an additional topological class, type II. Phenomenologically it is characterized by a parabolic spike frequency profile and undershooting spikes (Fig. 1B).

For this analysis we use the Chay-Cook- $(2,2) \operatorname{model} ; v$ and $n$ are fast variables, and $c$ and $s\left(\bar{\tau}_{\mathrm{s}}=10 \mathrm{sec}\right)$ are slow. The slow manifold is the set of equilibrium points of the fast subsystem (1-2), with $s$ and $c$ as parameters. However, the fast subsystem is now independent of $c$, which enters only through the function $s_{\infty}(v, c)$. Hence, we construct the bifurcation diagram in the $(s, v)$-plane rather than the $(c, v)$-plane (Fig. 12). The slow manifold is now $\mathrm{S}$-shaped rather than Z-shaped since $s$ is an excitatory variable; larger values of $s$ destabilize the resting solution. The periodic branch terminates at the knee in a SNIC bifurcation, so the fast subsystem is monostable.

The $s$ nullcline is now a two-dimensional surface, so in Fig. 12 we show projections for values of $c$ corresponding to the minimum and maximum taken on during a burst. For fixed $c$, the $v-n-S$ system can be bistable between silent and tonic spiking solutions, but it cannot alternate between the two. The root reason is that there is only positive feedback. Negative feedback is introduced by allowing $c$ to vary. This shifts the $s$ nullcline up and down so that the phase point is alternately attracted toward the silent and spiking states. During the silent phase, $c$ is near its minimum, and the slow nullcline lies below the manifold, so $\mathrm{d} s / \mathrm{d} t>0$ and the full-system trajectory travels rightward toward the spiking solution. When the phase point leaves the manifold and spiking is initiated the nullcline rises as $I_{\mathrm{Ca}}$, and thus $c$, increase. The nullcline eventually rises enough to change the direction of motion from rightward to leftward, moving the trajectory back toward the SNIC. When the SNIC is reached, the active phase ends and the phase point is attracted to the bottom branch of the manifold, where it continues to move leftward toward the stationary solution of the $v-n-s$ system. As it does so, $c$ decreases and the slow nullcline drops below the manifold, changing the direction of motion of the trajectory and 
restarting the oscillation. The model of Rinzel and Lee differs somewhat from Chay-Cook- $(2,2)$ in that the slow manifold, rather than the slow nullcline, is deformed by the second slow variable (when viewed in the $(x, v)$-plane).

In Fig. 13 we show a three-dimensional view of the trajectory in $(s, c, v)$ space. Since the slow manifold is independent of $c$, all planar slices perpendicular to the $c$ axis are identical. From this perspective the trajectory moves toward the viewer in the direction of increasing $c$ as it progresses from the beginning to the end of the active phase. Towards the end of this phase the spikes deform in the $s$-direction toward the knee and terminate. The trajectory then moves slowly along the bottom of the slow manifold in the direction of decreasing $c$, reinitiating the active phase when the knee is reached.

In Fig. 7 (with $\sigma=1-s$ ) this bursting oscillation is represented as a line segment from region $\mathrm{C}$ to the region on the other side of the $\mathrm{SN}$ curve in which a stable limit cycle encircles a single equilibrium point. When in region $\mathbf{C}$ the system is in a silent phase and becomes active when passage is made through the SNIC-curve to the adjacent region. Unlike types Ia, Ib, III, and IV, type II bursting traverses regions that are monostable.

The parabolic spike frequency profile of type II bursting results from the passage through a SNIC bifurcation both at the beginning and at the end of the active phase. Also, because of monostability, instantaneous perturbations

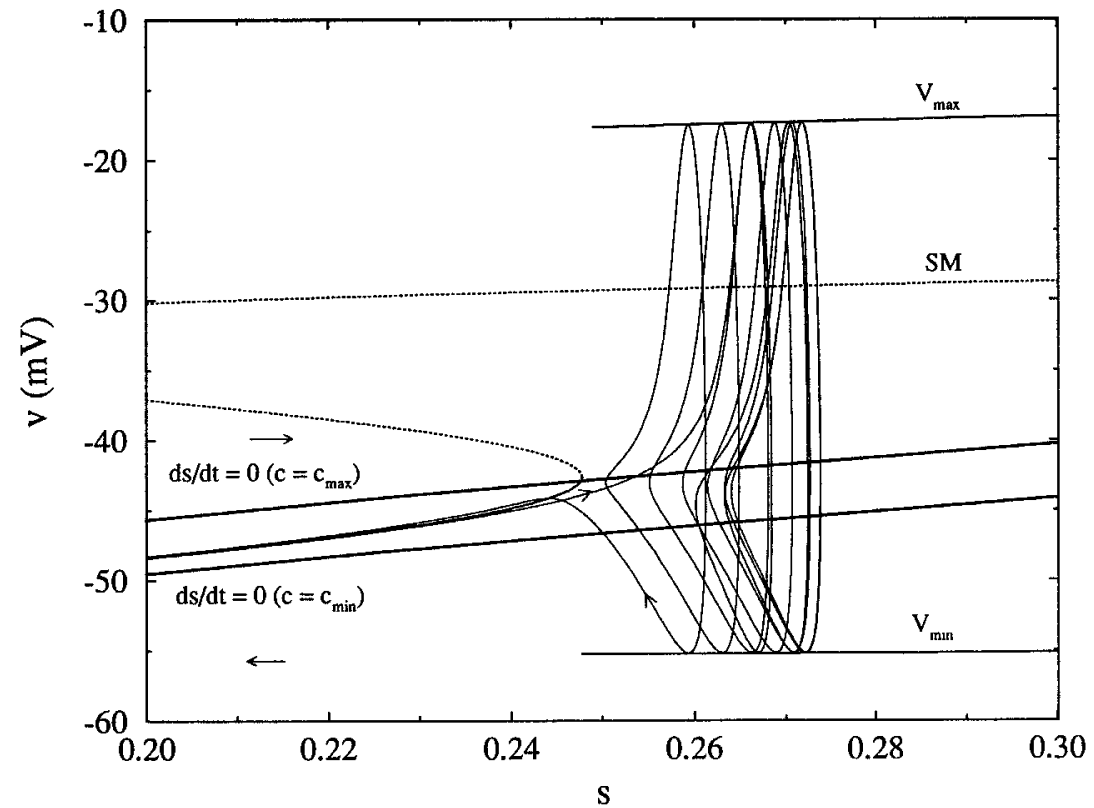

Figure 12. Dissection of type II bursting generated by Chay Cook- $(2,2)$ with $\lambda=0.6, f=0.0015, k_{\mathrm{c}}=0.03 \mathrm{~m} \mathrm{sec}^{-1}$ and $S_{\mathrm{s}}=10 \mathrm{mV}$. The two-dimensional slow nullcline is projected at $c=0.255 \mu \mathrm{M}\left(c=c_{\min }\right)$ and $c=0.375 \mu \mathrm{M}\left(c=c_{\max }\right)$. 
cannot reset the oscillation from a silent (active) to an active (silent) state. Finally, the spikes may undershoot the silent phase potential (Chay and Cook, 1988, Fig. 5).

When $\lambda$ is increased sufficiently, the periodic branch in Fig. 12 no longer terminates at the knee, but on the middle branch of the manifold. Because the fast subsystem is two-dimensional, there is no spike undershoot (Chay and Cook, 1988, Fig. 4). This is an example of type Ia bursting where, in Fig. 7, the endpoints of the representative line segment extend to the left of the SN curve and to the right of the SSL curve B due to the influence of the second slow variable which periodically varies the location of the slow nullcline. This type Ia oscillation differs from that described earlier in that the phase point first moves away from and then back toward the SL during the active phase, so the spike frequency profile is parabolic rather than monotonic. However, the parabolicity may be too subtle to be visible in the $v$ time course.

When $\lambda$ is decreased sufficiently, the periodic branch encloses all three branches of the slow manifold and the lower branch destabilizes via subcritical Hopf bifurcation, giving rise to an unstable periodic branch (as in Fig. 8B). When $\lambda=0.03$ the Hopf point lies beyond the left knee and the two periodic branches connect to form a single branch of periodics (Fig. 14B). This fast subsystem structure allows type III bursting. Indeed, bursting is generated even with $c$ fixed, since only one slow variable is necessary for this type of oscillation

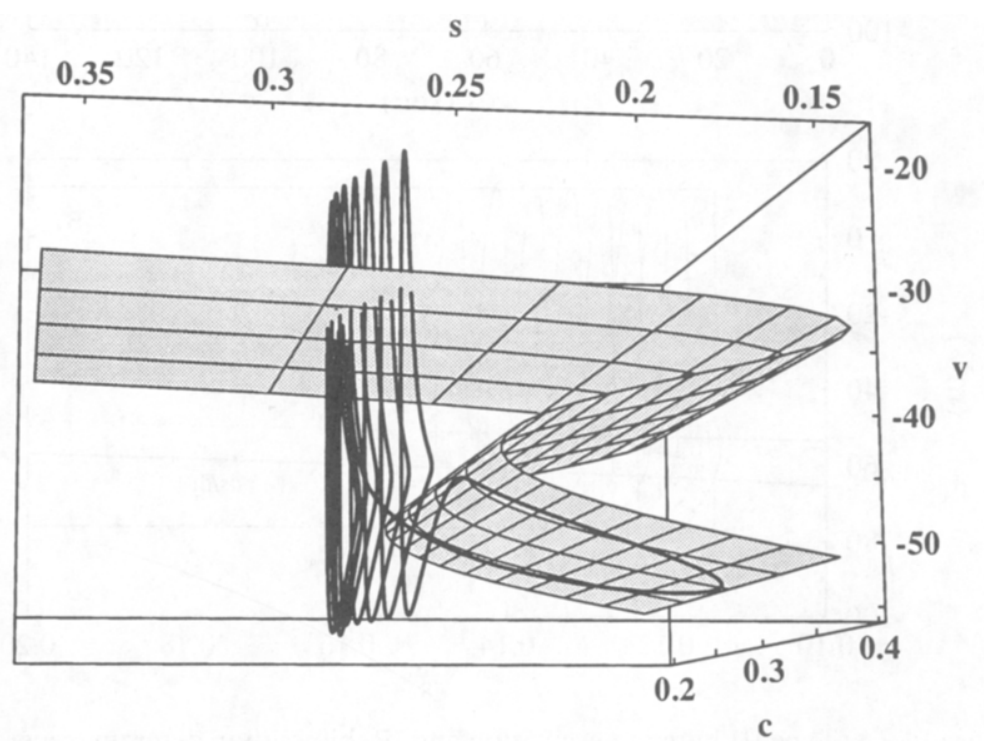

Figure 13. Three-dimensional view of the Chay-Cook- $(2,2)$ bursting dissected in Fig. 12. The slow manifold forms a surface, which has been shifted up slightly to make the entire bursting trajectory visible. 
(Fig. 14). This oscillation is similar to Fig. 6 of Chay and Cook (1988), which is also type III bursting driven by $s$, but with an inessential oscillation in $c$. The structure of Fig. 14B is identical to that of a model of cardiac ganglion cells of the lobster (Av-Ron et al., 1993, Fig. 4) and similar to the example of type III bursting due to Rinzel (1987, Fig. 4). Ours can be continuously deformed into the latter by varying a fast subsystem parameter that brings the knees of the slow manifold together in a cusp.

7. Discussion. We have described a classification scheme for bursting oscillations which extends the scheme of Rinzel (1987) and puts it in the context of a sequence of horizontal cuts through a two-parameter bifurcation diagram. In addition to the type Ia, II, and III oscillations described by Rinzel, we have
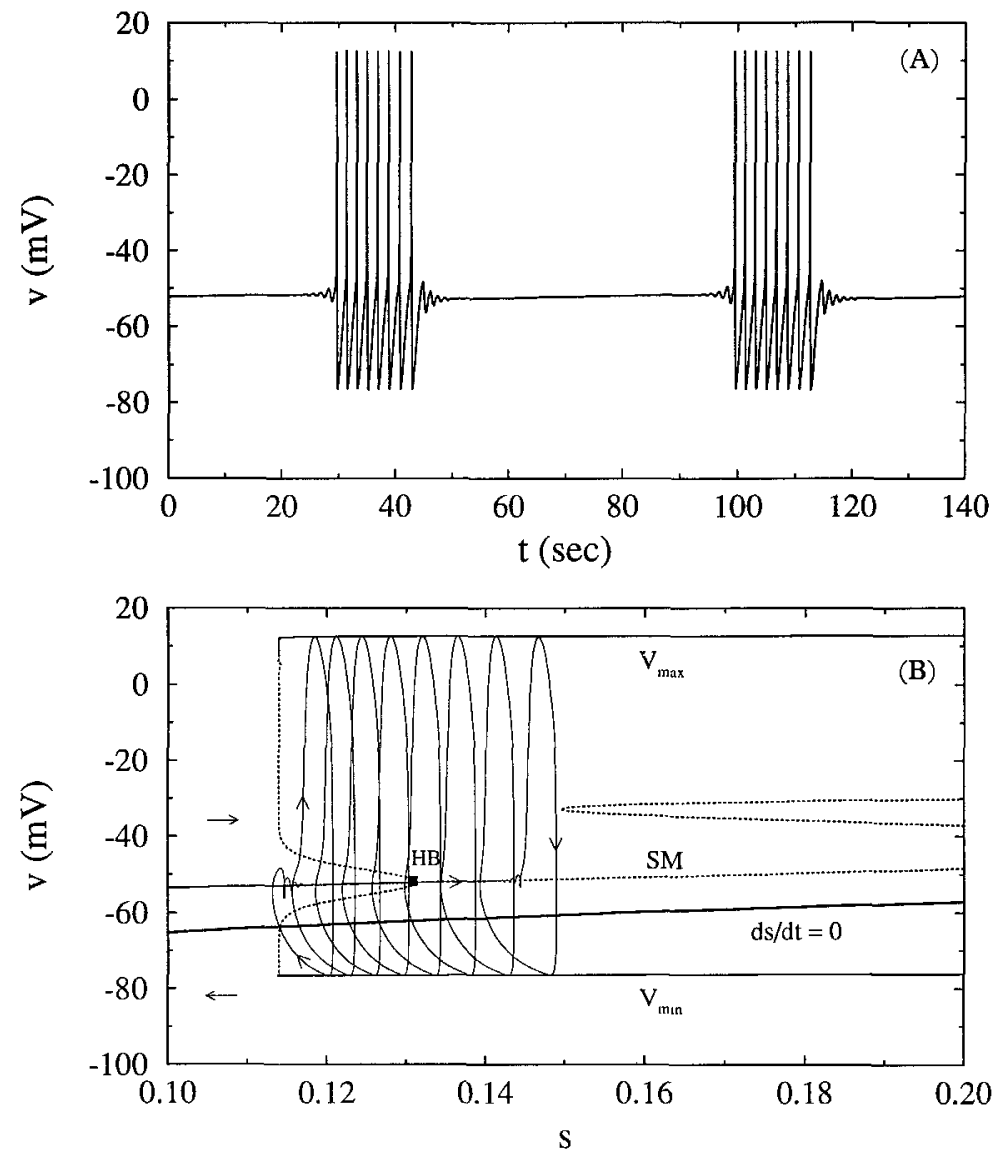

Figure 14. (A) Type III bursting oscillation and (B) bifurcation diagram, generated by the Chay-Cook model with $\bar{\tau}_{\mathrm{s}}=100 \mathrm{sec}, \lambda=0.03, S_{\mathrm{s}}=8 \mathrm{mV}$ and $c=0.13 \mu \mathrm{M}$. The small amplitude unstable and large amplitude stable periodic branches join to form a single branch of periodics. 
identified the "nearly" parabolic bursting of Pernarowski (1994, Fig. 1D) as a new type, Ib, and described an additional class, IV. Two additional oscillations, described in Fig. 1C, E of Pernarowski (1994) as "parabolic amplitude" and "tapered" bursting, are minor variants of type Ia. Parabolic amplitude bursting crosses the SH curve as it runs between the left SN and B (Fig. 7). This can only occur when the $\mathrm{SH}$ and left $\mathrm{SN}$ curves intersect at a value of $\mu$ below the maximum of curve $\mathbf{B}$. Tapered bursting can be visualized in Fig. 7 by moving the dashed type Ia line up so that it runs from the left to the right SN, passing through the SH curve once. Finally, the Smolen-Keizer bursting oscillation (Fig. 2B) has been identified as type Ia in spite of large undershooting spikes.

In our example, oscillations of type Ib and IV exist only over a small range of values of $\lambda$. This is a feature of the Chay-Cook model with a particular set of parameter values, and is not a general feature of bursting systems. Type $\mathrm{Ib}$ bursting, for example, is robust in Pernarowski (1994). Another modeldependent feature is that type III and IV bursting exist only for small values of $\lambda$, so that the time constant of the delayed rectifier is large. The time constant for the slow variable was increased so that the fast-slow dissection would be valid. Type III bursting, however, was demonstrated in Av-Ron et al. (1993) with a physiological delayed rectifier time constant of about $10 \mathrm{msec}$.

Local bifurcation theory suggests that the two-parameter bifurcation diagram of Fig. 7 occurs often in models of neuronal spiking. Specifically, the bifurcation diagram of Fig. 7 is found near a certain codimension-three bifurcation, a degenerate Takens-Bogdanov bifurcation of focus type (Dumortier et al., 1991). To find a focus TB bifurcation in a model with nullclines as in Fig. 5, first two parameters are varied so that the three fixed points come together in a cusp bifurcation. Then, the relative times scale of the two variables is adjusted so that the trace at the fixed point is zero. Finally, one must check that second and third order terms are within prescribed ranges, which is usually done through a normal-form calculation (Guckenheimer, 1986). Normal-form calculations on polynomial models show that the Hindmarsh-Rose fast subsystem (Hindmarsh and Rose, 1984), generalized to allow an arbitrary cubic nullcline and an arbitrary quadratic nullcline, has sufficient flexibility to exhibit a focus TB bifurcation, while the FitzHugh-Nagumo model (FitzHugh, 1961), with its cubic nullcline and linear nullcline, does not. Given their typical abundance of parameters, we expect that the fast subsystems of most bursting models can undergo a focus TB bifurcation and can therefore support all the types of bursting we have classified. However, the slow kinetics in a model may also have to be modified to go from one type of bursting to another.

Oscillations in most of the topological classes, types Ia, Ib, III and IV, can be generated by systems with a single slow variable due to bistability of the fast subsystem. Only oscillations of type II require two slow variables, which must 
interact with the fast subsystem to generate an oscillation on a slow time scale, driving the fast subsystem back and forth through a silent and an active phase. Because the fast subsystem in type II is monostable, while it is bistable in all other classes, it would seem that this distinction could be used in an experimental setting to aid in the classification of a bursting oscillation. Such a classification is helpful if one wishes to construct a model of bursting in an excitable membrane, where membrane potential is quite often the only observable. One could perturb the system, by applying a brief current pulse through the membrane, and if the oscillation cannot be reset from its active (silent) to silent (active) phase the bursting is of type II. However, the converse is not true. Examples exist of models generating type II bursting in which instantaneous perturbations can reset the oscillation. These include the model of bursting in $R_{15}$ by Bertram $(1993,1994)$, where one of the slow variables is sufficiently fast at some voltages to respond quickly to the perturbation (although this model does not fit precisely into the present framework since it has a quintic, rather than cubic, slow manifold), and the $R_{15}$ model by Canavier et al. (1991) in which several bursting, beating, and chaotic attractors coexist (Canavier et al., 1993). Phase resetting, then, provides only a limited experimental tool in the classification of a bursting oscillation.

Other experimentally observable features are the spike frequency profile and the lack or presence of spike undershoot. While the type Ia frequency profile is monotonic when the generating system contains one slow variable (this is strictly true only near the homoclinic orbit, though the authors are not aware of any example where it is not true for the entire active phase), type Ia bursting can be generated by a system with two slow variables, in which case the frequency profile may be parabolic. Type II bursting is parabolic, but may appear monotonic since the passage through the SNIC is often fast at the beginning of the active phase. Finally, the active phase of type III and IV bursting is neither initiated nor terminated by a homoclinic bifurcation, so the spike frequency profile may be monotonic, parabolic, or neither. In any case, the monotonicity or parabolicity of an oscillation may be too subtle to discern, particularly in the presence of noise.

The lack or presence of spike undershoot is also a limited indicator of topological class. While type Ia oscillations do not display spike undershoot if the fast subsystem is two-dimensional, in higher dimensions undershoot may be present. Adding to the uncertainty, the dimension of the fast subsystem is typically not known in an experimental setting. On the other hand, while type II oscillations always exhibit undershoot, spikes may not undershoot the silent phase voltage, giving the appearance of a voltage plateau (Rinzel and Lee, 1987, Fig. 7). We do observe, however, that in type III and IV bursting spikes always undershoot the silent phase voltage. Hence, oscillations without this property must be of one of the other types. 
Another property which sets type III and IV bursting apart from the others is the presence of small oscillations before and after (type III) or after (type IV) the active phase. If, however, the subcritical Hopf point is close to the knee, small oscillations may not be present before the active phase of type III bursting. Even if present, such small oscillations may be obscured by noise and thus hard to detect.

The topological classification scheme presented here encompasses a wide variety of bursting oscillations. However, there are some bursting models that do not fit into the scheme. They include the Smolen-Sherman model (Smolen and Sherman, 1994), which has two "slow" variables, though only one is slow at any given time, and the models of Bertram (1993) and Rush and Rinzel (1994) which have quintic, rather than cubic, slow manifolds (see also Wang and Rinzel (1994)). Our classification scheme is developed around the simplest biophysical burster models and may not apply to more complex systems, where network or other effects may be responsible for driving the burst.

T.K. was supported by NIMH grant MH-44809 and NIH grant NS-16803 to Avis Cohen.

\section{REFERENCES}

Alving, B. 1968. Spontaneous activity in isolated somata of Aplysia pacemaker neurons. J. Gen. Physiol. 51, 29-45.

Ashcroft, F. and P. Rorsman. 1989. Electrophysiology of the pancreatic $\beta$-cell. Prog. Biophys. molec. Biol. 54, 87-143.

Av-Ron, E., H. Parnas and L. Segel. 1993. A basic biophysical model for bursting neurons. Biol. Cybern. 69, 87-95.

Baer, S. M., T. Erneux and J. Rinzel. 1989. The slow passage through a Hopf bifurcation: delay, memory effects, and resonance. SIAM J. Appl. Math. 49, 55-71.

Bertram, R. 1993. A computational study of the effects of serotonin on a molluscan burster neuron. Biol. Cybern. 69, 257-267.

Bertram, R. 1994. Reduced-system analysis of the effects of serotonin on a molluscan burster neuron. Biol. Cybern. 70, 359-368.

Canavier, C. C., J. W. Clark and J. H. Byrne. 1991. Simulation of the bursting activity of neuron $\mathrm{R}_{15}$ in Aplysia: role of ionic currents, calcium balance, and modulatory transmitters. $J$. Neurophysiol. 66, 2107-2124.

Canavier, C. C., D. A. Baxter, J. W. Clark and J. H. Byrne. 1993. Nonlinear dynamics in a model neuron provide a novel mechanism for transient synaptic inputs to produce long-term alterations of postsynaptic activity. J. Neurophysiol. 69, 2252-2257.

Chay, T. R. and D. L.Cook. 1988. Endogenous bursting patterns in excitable cells. Math. Biosci. 90, 139-153.

Crunelli, V., J. S. Kelly, N. Leresche and M. Pirchio. 1987. The ventral and dorsal lateral geniculate nucleus of the rat: intracellular recordings in vitro. J. Physiol. 384, 587-601.

Dean, P. M. and E. K. Matthews. 1970. Glucose-induced electrical activity in pancreatic islet cells. J. Physiol. 210, 255-264.

Deschênes, M., J. P. Roy and M. Steriade. 1982. Thalamic bursting mechanism: an inward slow current revealed by membrane hyperpolarization. Brain Res. 239, 289-293. 
Doedel, E. 1981. Auto: A program for the automatic bifurcation analysis of autonomous systems. Cong. Num. 30, 265-284.

Dumortier, F., R. Roussarie and J. Sotomayor. 1991. Generic 3-parameter families of planar vector fields, unfoldings of saddle, focus and elliptic singularities with nilpotent linear parts. In Bifurcations of Planar Vector Fields: Nilpotent Singularites and Abelian Integrals, F. Dumortier, R. Roussarie, J. Sotomayor and H. Żoladek (Eds), Lecture Notes in Mathematics, Vol. 1480, pp. 1-164. Berlin: Springer.

Ermentrout, G. B. and N. Kopell. 1986. Parabolic bursting in an excitable system coupled with a slow oscillation. SIAM J. Appl. Math. 46, 233-253.

FitzHugh, R. 1961. Impulses and physiological states in theoretical models of nerve membrane. Biophys. J. 1, 445-466.

Gear, C. 1967. The numerical integration of ordinary differential equations. Math. Comp. 21, $146-156$.

Guckenheimer, J. 1986. Multiple bifurcation problems for chemical reactions. Physica 20D, $1-20$.

Guckenheimer, J. and P. Holmes. 1983. Nonlinear Oscillations, Dynamical Systems and Bifurcations of Vector Fields, pp. 353-423. Berlin: Springer.

Guckenheimer, J., S. Gueron and R. M. Harris-Warrick. 1993. Mapping the dynamics of a bursting neuron. Phil. Trans. Roy. Soc. Lond. 341, 345-359.

Harris-Warrick, R. M. and R. E. Flamm. 1987. Multiple mechanisms of bursting in a conditional bursting neuron. J. Neurosci. 7, 2113-2128.

Hindmarsh, A. 1974. An ordinary differential equation solver. Technical report UCID-30001, Lawrence Livermore Laboratory.

Hindmarsh, J. L. and R. M. Rose. 1984. A model of neuronal bursting using three coupled first order differential equations. Proc. $R$. Soc. Lond. B 221, 87-102.

Hudson, J. L., M. Hart and D. Marinko. 1979. An experimental study of multiple peak periodic and nonperiodic oscillations in the Belousov-Zhabotinskii reaction. J. Chem. Phys. 71, 1601-1606.

Johnson, S. W., V. Seutin and R. A. North. 1992. Burst firing in dopamine neurons induced by N-Methyl-D-Aspartate: role of electrogenic sodium pump. Science 258, 665-667.

Pernarowski, M. 1994. Fast subsystem bifurcations in a slowly varying Liénard system exhibiting bursting. SIAM J. Appl. Math. 54, 814-832.

Plant, R. E. and M. Kim. 1976. Mathematical description of a bursting pacemaker neuron by a modification of the Hodgkin-Huxley equations. Biophys. J. 16, 227-244.

Rinzel, J. 1985. Bursting oscillation in an excitable membrane model. In Ordinary and Partial Differential Equations, B. D. Sleeman and R. J. Jarvis (Eds), Lecture Notes in Mathematics, Vol. 1151, pp. 304-316. Berlin: Springer.

Rinzel, J. 1987. A formal classification of bursting mechanisms in excitable systems. In Mathematical Topics in Population Biology, Morphogenesis and Neurosciences, E. Teramoto and M. Yamaguti (Eds), Lecture Notes in Biomathematics, Vol. 71, pp. 267-281. Berlin: Springer.

Rinzel, J. and Y. S. Lee. 1987. Dissection of a model for neuronal parabolic bursting. J. Math. Biol. 25, 653-675.

Rinzel, J. and W. C. Troy. 1982. Bursting phenomena in a simplified Oregonator flow system model. J. Chem. Phys. 76, 1775-1789.

Rush, M. E. and J. Rinzel. 1994. Analysis of bursting in a thalamic neuron model. Biol. Cybern. 71, 281-291.

Schecter, S. 1987. The saddle node separatrix loop. SIAM J. Math. Anal. 18, 1142-1156.

Sherman, A. and J. Rinzel. 1992. Rhythmogenic effects of weak electrotonic coupling in neuronal models. Proc. Natl. Acad. Sci. USA 89, 2471-2474.

Smolen, P. and J. Keizer, 1992. Slow voltage inactivation of $\mathrm{Ca}^{2+}$ currents and bursting mechanisms for the mouse pancreatic beta-cell. J. Membrane. Biol. 127, 9-19.

Smolen, P. and A. Sherman. 1994. Phase-independent resetting in relaxation and bursting oscillators. J. Theor. Biol. 169, 339-348. 
Strumwasser, F. 1967. Types of information stored in single neurons. In Invertebrate Nervous Systems: Their Significance for Mammalian Neurophysiology, C. A. G. Wiersma (Ed.), pp. 290-319. Chicago: The University of Chicago Press.

Traub, R. D., R. K. S. Wong, R. Miles and H. Michelson. 1991. A model of a CA3 hippocampal pyramidal neuron incorporating voltage-clamp data on intrinsic conductances. J. Neurophysiol. 66, 635-650.

Wang, X.-J. and J. Rinzel. 1994. Oscillatory and bursting properties of neurons. In The Handbook of Brain Theory and Neural Networks, M. A. Arbib (Ed.). Cambridge, MA: The MIT Press.

Wang, X.-J., J. Rinzel and M. A. Rogawski. 1991. A model of the T-type calcium current and the low-threshold spikes in the thalamic neurons. J. Neurophysiol. 66, 839-850.

Wong, R. K. S. and D. A. Prince. 1981. Afterpotential generation in hippocampal pyramidal cells. J. Neurophysiol. 45, 86-97.

Received 31 March 1994

Revised version accepted 19 September 1994 